\title{
The Endothelial Barrier Restricts Endocrine Actions to the Luminal Vascular Receptors: Changing the Paradigm: A Didactic Approach
}

\author{
Rafael Rubio
}

\section{ABSTRACT}

In 1849, the first list of endocrine hormones was discovered and proposed that the synthesizing gland delivers it to the circulation. The circulatory hormone reaches the target organ, physically unimpeded acts directly on the parenchymal cells. Such a simplistic view persists despite new knowledge of an endothelial wall barrier and implications for every parenchymal cell in the body. This misconception leads to inadequate interpretations of data, wrong diagnosis and therapeutic expectations, erroneous hypotheses, and misleads further research work. The quest of this review is to play down this misconception by pointing out key overlooked findings of the vascular endothelial wall: 1) The selective endothelial barrier physically separates two same-hormone-containing compartments; the endocrine and the interstitial autocrine hormone compartments, 2) the hormone concentrations values in these compartments are independent of each other, 3) in each compartment the hormone acts solely on the receptors of that particular compartment, 4) multiple intravascular endocrine hormones act solely on their corresponding luminal endothelial membrane receptor (LEMR), without directly acting on the parenchymal cells, 5) Agonist-activation of LEMR triggers the release of specific paracrine endothelial agents that in conjunction with autocrine interstitial hormone modulate parenchymal function(s) and perhaps the turnover of the interstitial autocrine hormone, 6) these hormone compartments, functionally interact via paracrine exchange signaling, and the integrated intercourse of all these events result in the final hormonal organ effect. The present challenges to achieving more rationale therapeutic effects are to design agonists or antagonists that exclusively gain access to a target compartment and have high specificity for the receptor of the cells in that compartment.

Keywords: Autocrine hormones, organ compartments, paracrine signaling.
Submitted : September 18, 2021

Published : November 13, 2021

ISSN: $2593-8339$

DOI: $10.24018 /$ ejmed.2021.3.6.1070

\section{R. Rubio*}

Emeritus Prof., Department of Molecular Physiology and Biological Physics, School of Medicine, University of Virginia, Charlottesville, USA.

(e-mail: rrubio@uaslp.mx)

*Corresponding Author

\section{INTRODUCTION}

\section{A. The Old Delusion Model: Implicit Assumptions}

The 1829 observations by Arnold Adolf Berthold proposed that testicles release active principles into the circulation that define male's masculinity [1]. In more than 150 years, have been discovered many other distinct "endocrine hormones or cytokines" secreted into the circulation. The proposed concept still in use was that any of these vascular circulating active substances reach a target organ(s) (black triangles, Fig. 1). After that, the incoming endocrine hormone unimpeded crosses the inert vascular endothelial wall, diffuse freely into the interstitium, and reaches other organ compartments. It acts directly and solely on cell membrane receptors of the constituting parenchymal cells of all compartments altering their metabolism and function, "the response."

The affected cell is various:

1) contraction for vascular smooth muscle in the case of blood vessels,
2) contraction and electric properties for the miscellaneous cardiac myocytes in the heart,

3 ) cardiac and skeletal muscle glucose consumption,

4) transmitter release of the nerve end terminals,

5) ion or water transfer for tubular epithelial cells in the kidney, and mRNA activation,

6) epinephrine, and steroid secretion for medullary and cortical cells respectively, in the adrenal gland, among many other examples.

This persistent misconception has relevant implications; for example, it is behind the rationales for the therapeutic hormone treatments and the studies on parenchymal hormone-receptor mechanisms. This review addresses the reasons why we must reevaluate this concept. 


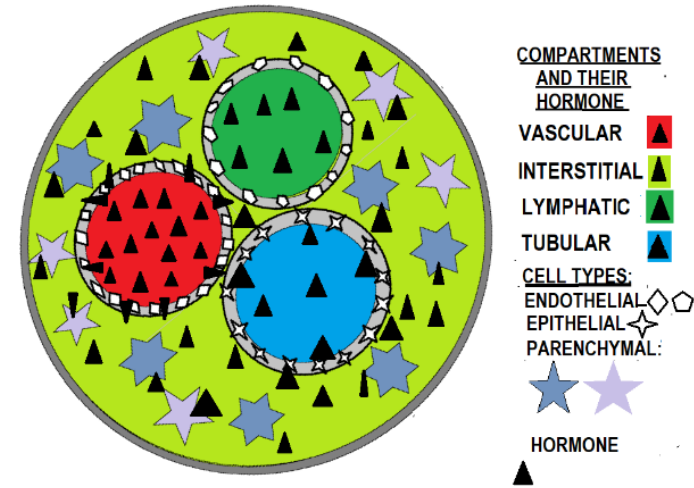

Fig. 1. This cartoon represents the current frequently accepted model for endocrine action. It represents a cross-section of an organ and its compartments; intravascular (red), interstitial (light green), tubular (blue), and lymphatic (green). The wall of each chamber is made of specialized cells either, endothelial, epithelial, or secretory. In this case, the circulating intravascular hormone (black triangles) exchanges freely across an inactive endothelial wall. It has direct access everywhere to the hormone receptors of all cell types in the parenchyma.

\section{IDENTIFICATION OF THE PROBLEM}

Our excursion into this problem originated during our early studies on adenosine's mechanisms of action on the functions of diverse cardiac myocytes. We assumed that a circulating agonist directly accessed membrane receptors of all parenchymal cells (Fig. 1). However, when further experimental evidence accumulated together with studies on the effects of other hormones, we found that this simple model is inaccurate and should be discarded [2]-[26]. The basis for this denunciation is in sections IIA and IIB.

\section{A. LACK of Hormone-Free Exchange between Circulatory and Interstitial Compartments, Diffusional Studies}

1) Agonists, Either Large or Small-Sized, Have the Same Potency and Speed of Action

The diffusion rates and space/second occupied by a solute in a free diffusion system varies inversely to their size. Intravascular agonists that are 100 times the size of their natural agonists have a lower action speed and potency. Unexpectedly, their effects being either: coronary tone, inotropism, dromotropism, spontaneous ventricular rhythm, bradycardia, skeletal muscle vascular tone, and enhanced glucose consumption; they act as fast and potent as the smallsize agonist [2]-[19], [23]-[25]. The fact that intravascular large-size agonists and their smaller counterparts behave equally suggests that both act at the same site and at the same speed because they do not diffuse across the endothelial wall. The diverse responses: coronary tone, inotropism, dromotropism, spontaneous ventricular rhythm, bradycardia, skeletal muscle vascular dilation and enhanced glucose consumption, Acetylcholine release, are cell responses of coronary smooth muscle, ventricular muscle myocytes, auricular-ventricular nodal cells, ventricular Purkinje cells, sinus nodal cells, skeletal muscle; arterial vascular smooth muscle, cardiac muscle glucose consumption, and vagal nerve endings. Quite a diversity of hormones, parenchymal cells, and functions [2]-[19], [23]-[26].

2) There Are Two Hormonal Pools: A Circulating Endocrine Pool and An Interstitial Autocrine Pool
The advent of sample the interstitial fluid in various organs contained several hormones and the Adenosine, Angiotensin II, or Bradykinin concentrations determined [27]-[37]. The endocrine and the autocrine pools of these hormones are physiologically active. In a freely diffusing system, they would exchange tending to an equilibrium, i.e., to equalize their concentration (Fig. 2). i.e., there would have to exist a proportionality.

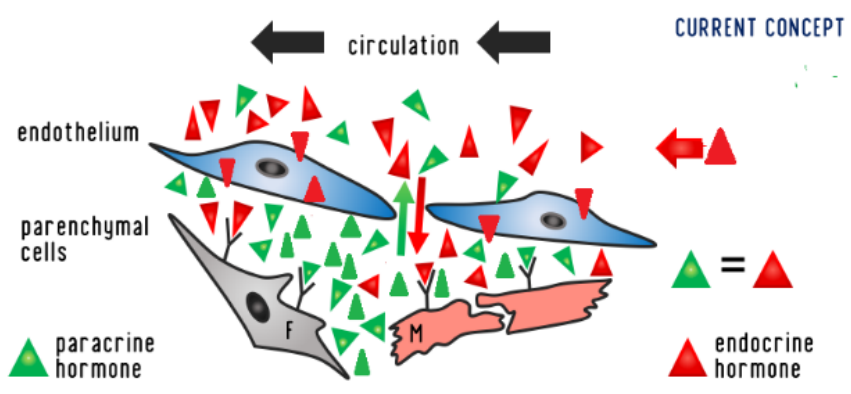

Fig. 2. This simpler scheme illustrates a cross-section of a hypothetical organ. Hormone freely exchanging across an inactive endothelial wall. Red triangles represent the circulating intravascular hormone which is also present interstitially. Nevertheless, it is of autocrine origin, and its concentration is greater than the vascular (green triangles). At least two compartments contain the same hormone: intravascular and interstitial compartments (27-37). F and M represent parenchymal cells.

Fig. 3A and 3B illustrate what would happen to the intravascular or interstitial hormone concentrations in a freely diffusing exchange system when changed. Figure 3A shows the case when the intravascular concentration is greater than the interstitial, causing it to rise. In contrast, when the incoming circulation has $\mathrm{a} \approx$ zero endocrine concentration, a washout of the interstitial endocrine should decrease its concentration (Fig. 3B). The interstitial and circulatory hormone concentrations tend to equilibrium and always showing a proportionality.

There is no equilibrium between interstitial and intravascular hormone concentrations. The interstitial is up to a hundred times greater for either adenosine, Ang II, or bradykinin. Moreover, an intravascular infusion of these hormones at concentrations several orders of magnitude greater than the interstitial does not alter the interstitial concentration [27]-[37]. These facts indicate that a selective physical barrier minimizes free diffusion between the interstitial and intravascular hormone pools.

\section{3) Circulatory and Interstitial Autocrine/Endocrines Pools}

\section{Do not Exchange Freely}

Thus, circulating hormones do not freely traverse the endothelial wall into the interstitium. They cannot act solely and directly on parenchymal cells. Then, if circulating hormones do not have direct access to parenchymal cells, if it does not change the interstitial concentration, how activation takes place when they are not present? How does the organ, any organ respond to the circulating endocrine hormone?

Figure 3 illustrates the implications of a hormone freely exchanging bi-directionally across an inactive endothelial wall. 


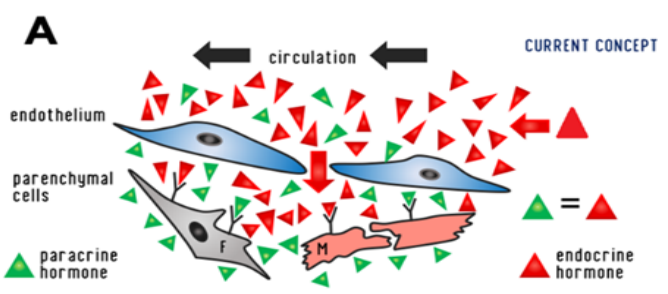

B

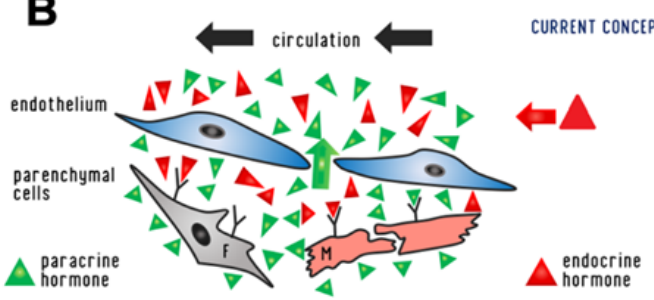

Fig. 3A. Shows that perfusion with a high concentration of endocrine hormone (red triangles) elevates its diffusion (red arrow) into the interstitium, raising the interstitial endocrine concentration.

Fig. 3B. Shows that perfusion with a negligible concentration of endocrine hormone (red triangles) elevates diffusion from the interstitium (green arrow). The washout of the interstitial autocrine hormone reduces the interstitial hormone concentration (green triangles).

\section{4) Alternative Hypothesis}

A circulating hormone solely acts on endothelial luminal membrane receptors located along the vascular tree. The endothelium, in turn, releases diverse paracrine agents that target a particular parenchymal response.

The presence of hormone receptors in the luminal endothelial surface of blood vessels and their selective activation were elegantly established in the early 1970s by Armstrong et al. for insulin [2] and Bevan and Duckles for $\alpha$ adrenergic receptors [11]. After that, Furchgott and Zawadski [38] show that the relaxation of arterial smooth muscle induced by acetylcholine requires endothelial cells. An effect mediated by the endothelial paracrine release of nitric oxide, one of the many bioactive agents that the endothelium can release [4], [39].

To test the proposed hypothesis in vivo, one can use:

1) agonists and antagonists so large that they cannot permeate the endothelial wall when administered. Being confined intravascularly restricts their action to the luminal endothelial receptors,

2) The receptor protein must be present in the luminal endothelial membrane.

\section{B. Lack of Hormone Exchange between Circulatory and Interstitial Compartments, Functional Studies}

1) Synthesis of Large Size Agonists and Antagonists; Diameter 70-120 nm

Extensive studies on the passive exchange diffusion of inactive solutes across the vascular wall have established that the upper dimensional limit of capillary pore diameter is 24$32 \mathrm{~nm}$. Meaning that any molecule bigger than $32 \mathrm{~nm}$, regardless of its chemical nature, will not passively cross the endothelial barrier [41]. We synthesized; via covalent bonds, agonists, and antagonists to large molecular weight, nobioactive matrices with a molecular diameter between 70 to $120 \mathrm{~nm}$ [42]. These significant size agonists or antagonist's polymers, remains in the vessel lumen when intravascularly perfused, where they can solely exert their action (Fig. 4). We control that an effect from the release of free small size agonist or antagonist from the large matrix did not occur. Simply the molecular complex does not hydrolyze. The following hormones, most of their antagonists and their corresponding large-size molecules: Adenosine A1, A2, and A3 agonists, acetylcholine, $\alpha$-adrenergic, Bradykinin, Insulin, Vasopressin, Substance P, Testosterone, Estradiol, and Angiotensin II were studied [2], [3]-[7], [12]-[19], [23][25], [34].

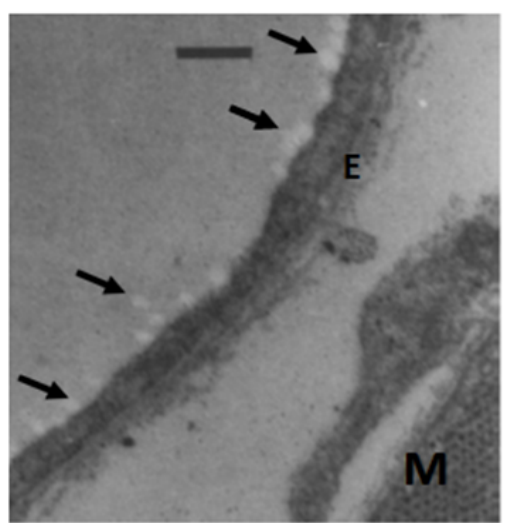

Fig. 4. Electron micrograph shows a microbead-agonist complex (diameter $\approx 80 \mathrm{~nm}$ ) lining a capillary's intravascular endothelial surface (E). The spheres appear as pale dots (arrows) against the endothelial luminal membrane. In the lower right corner, there is a portion of a myocyte (M). Notice the thick myofilaments [3],[4].

\section{2) The Endothelial Luminal Membrane of Many Organs Can Be Reliably Isolated}

Receptor-proteins for several hormones are present in the endothelial luminal membrane. Moreover, in the luminal endothelial membrane, the presence of at least fourteen receptor-proteins has been established: Angiotensin AT1 and AT2, insulin, endothelin, prolactin, vasopressin, thromboxane $\mathrm{A} 2$, adenosine $\mathrm{A} 1, \mathrm{~A} 2$, and $\mathrm{A} 3$, bradykinin, adrenergic $\alpha, \beta 1$, and $\beta 3$ are present [12]-[14], [17], [43]-[45]. Fig. 5 shows an example of the presence of these proteins.

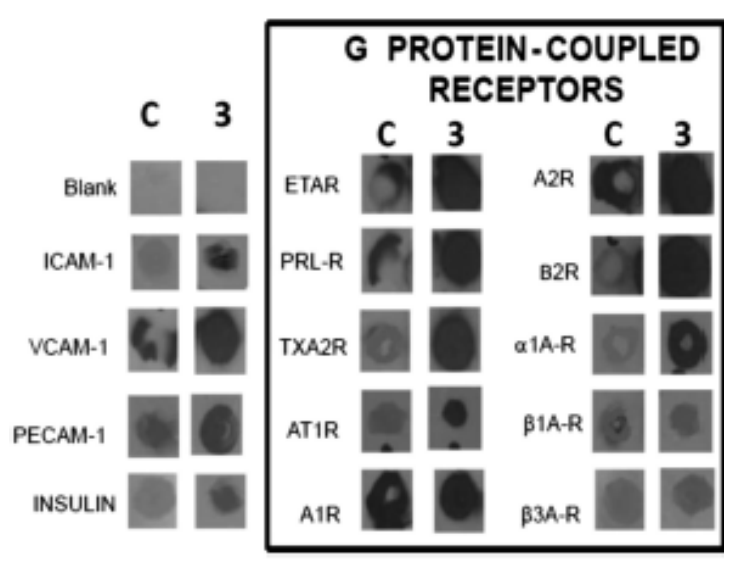

Fig. 5. Immune dot-blots of various proteins in the coronary luminal endothelial membrane. Column $\mathrm{C}$ and Column 3 are the same protein in the original fraction. From left to right; blots in the left and outside the box and in descending order, the two columns of immune dots, show the Blanks, with no reaction background, and three selectins ICAM-1, VCAM-1, PECAM-1, and the insulin receptor. The four columns inside the box correspond to ten $\mathrm{G}$ protein-coupled receptors ETAR, PRL-R, TXA2R,

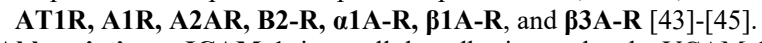

Abbreviations. ICAM-1; intercellular adhesion molecule, VCAM-1;

vascular cell adhesion molecule, PECAM-1; platelet endothelial cell adhesion molecule, ETAR; endothelin receptor, PRLR; prolactin receptor,

TXA2R; thromboxane A2 receptor, AT1R; ANG II receptor, A1R; and A2R; adenosine receptors, B2R; bradykinin receptor, $\boldsymbol{\alpha 1 A - R , ~} \boldsymbol{\beta 1 A}-\mathbf{R}$, and B3A-R; adrenergic receptors. 


\section{3) Effects of a Circulating Agonist and Its Large Size- Agonist}

Let us assume that the vascular endothelial wall is freely permeable to an agonist (the traditional point of view), and its actions restrict to the organ's parenchymal cells. The arterially administered agonist crosses the endothelial wall. It directly accesses parenchymal cell membrane receptors inducing a cellular response (Fig 6A, expected response). In contrast, the arterially administered large size-agonist does not cross the endothelial wall Fig 6B). It does not access the parenchymal membrane receptors, and no response occurs (Fig 6B, expected response). Still, a parenchymal response by the large size-agonist occurs and is equal to that caused by the freely diffusible agonist (Fig 6B, actual response). Here, the necessary conclusion is that the small and large size agonists must act at an exact location; the membrane receptors located on the endothelial luminal membrane.
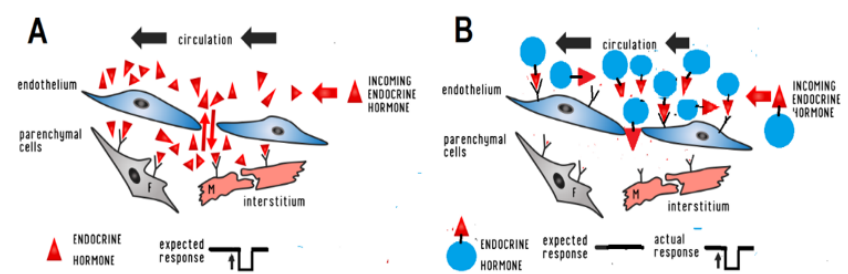

Fig. 6. Tissue distribution of (A) small permeable agonist and (B) the large size impermeable agonist when arterially given (upper drawings). The lower traces show the expected and the actual corresponding parenchymal responses. In these two cartoons, since the point is to analyze whether the intravascular hormone is or is not the sole direct stimulus of the observed response, the endogenous hormone has not been drawn. Upper drawings:

Fig 6A illustrates the small size agonist (red triangles) that crosses the inactive endothelial wall and acts on the parenchymal cell receptors (Y), triggering the expected response (lower trace). Fig. 6B illustrates that the large size-agonist (sphere with a red triangle) does not cross the endothelial wall and does not act on the parenchymal cell receptors (Y), not triggering an expected response (left lower trace). However, an actual response occurs (right lower traces), implying it only acts on luminal endothelial receptors

(Y). This response is the same magnitude as the small agonist due to activating receptors on the luminal endothelial membrane [2]-[7], [11]-[19], [23]-[25], [34].

4) Agonist and Its Large Size-Agonist When Intravascularly Administered Together, at the Receptor Site, Compete with Each Other

Upon acting on its receptor, an agonist triggers two mechanisms: receptor activation and its internalization [12], [17], [18], [46]-[50]. During sustained agonist administration, the receptor membrane density gradually decreases, internalization. Receptor internalization results in a parallel decrease of the physiological response; desensitization (Section IIB-6). The two agonists, Ang II and Ang II-polymer (Ang-Pol), activate the luminal endothelial receptor equally. However, each agonist induces a quantitatively distinct receptor internalization and desensitization [12], [13], [17], [18]. Desensitization is rapid for Ang II and slow for the Ang II polymer. However, when simultaneously administered Ang II and Ang II-Pol, desensitization rate of a value in between Ang II's and Ang II-Pol's rates. The concentrations ratio [Ang II]: [Ang II-Pol] determines the desensitization rate. At a ratio 1:0 (only Ang II present) or 0:1 (only Ang IIPol present), the desensitization rates are Ang II's and Ang II-Pol's, respectively. The rate decreases as [Ang II]: [Ang IIPol] drops, and at a ratio $\geq 1: 3$, (1 Ang II +3 Ang II-Pol) the rate is equal to that of Ang II-Pol alone. These results show that Ang II and Ang II-Pol compete for binding in the endothelial luminal membrane AT1R [17], [18].

5) Blockade of the Effects of a Circulating Agonist by a Small Size Diffusible Antagonist and by a Bulky Antagonist

Let us continue with the assumption (the traditional point of view) that the vascular endothelium is freely permeable to an agonist and antagonist. Simultaneously arterially administered, agonist and antagonist cross the endothelial wall. Both reach the parenchymal cell receptors, competing for binding to it, and the antagonist can block the agonist effect (Fig. 7A, expected response). In contrast, the agonist and the bulky antagonist simultaneously cross the endothelial wall, and the massive antagonist remains intravascularly (Fig. 7B). Upon reaching the parenchymal cell receptors, the agonist causes a response because the bulky antagonist is absent (Fig. 7B, expected response). However, a response does not occur. It is blocked (Fig. 7B, actual response). Here again, the conclusion is that the small agonist, the tiny antagonist, and the massive size antagonists cannot be considered "diffusible" and must compete for binding to membrane receptors at the same site, the endothelial luminal membrane.
A

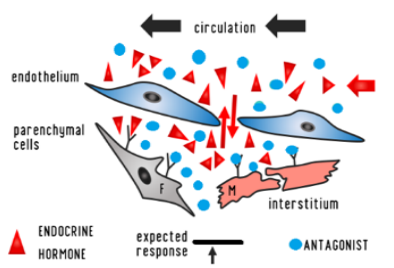

B

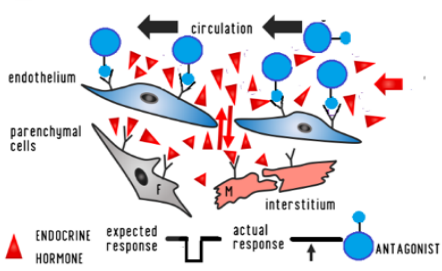

Fig. 7. Tissue distribution of (A) permeable antagonist and agonist and (B) impermeable antagonist and permeable agonist when arterially administered. The lower traces show the predicted and actual parenchymal responses. In these two cartoons, since the point is to analyze whether the intravascular hormone is or is not the sole direct stimulus of the observed response, the endogenous hormone has not been drawn. Fig. 7A. Illustrates the arterial infusion of a small sizes agonist (red triangles) and antagonist (blue spheres), both cross the endothelial wall and compete for binding to the parenchymal cell receptors (Y). Because the antagonist blocks the parenchymal receptor, there is not the expected response (lower trace). Fig. 7B. Illustrates the arterial infusion of a small-sized agonist (red triangles) and large-size-antagonist (large and small purple spheres), which remains intravascularly. The permeable agonist (red triangles) crosses the endothelial wall, binds to the parenchymal receptors without competition from the antagonist, and generates the expected response (left lower trace). However, the response is blocked (right, actual response) because hormone activation of receptors occurs only in the luminal endothelial membrane (Y), [4], [6], [7], [14], [16]-[18], [26], [34].

6) Only a Vascular Antagonist Blocks Vascular Agonist Effects, While Interstitially, the Antagonist Has No Action

Reference [26], implanting a micro-dialysis device in the heart, measured interstitial acetylcholine released by electrical stimulation of vagal nerve endings. Ang II intravascular administration inhibits Acetylcholine release, and intravascular losartan blocks this effect. However, losartan interstitial delivery does not block intravascular Ang II action because endocrine Ang II is absent interstitially. Alternatively, if Ang II is interstitially present, it does not affect acetylcholine releasing nerve terminal. However, ATP is likely the endothelial paracrine mediator [13], inhibiting cholinergic nerve endings [72].

In summary, the following membrane receptors are present in the endothelial lumen: Angiotensin AT1 and AT2, insulin, 
endothelin, prolactin, vasopressin, thromboxane $\mathrm{A} 2$, and adenosine A1, A2, and A3, bradykinin, adrenergic $\alpha, \beta 1$, and $\beta 3$ [12]-[14], [18], [43]-[45]. These and other luminal receptors can be hormone-activated or block. Their activation triggers the release of specific endothelial paracrine agents, causing a specific parenchymal response [2], [3], [5]-[7], [11], [13], [16], [18], [23]-[25], [45], [51]. In response to hormone activation in vivo, few paracrine endothelial agents, so far well-identified, are Nitric oxide, ATP, Prostaglandins, Thromboxane A2, and likely ChiroInositol [5]-[7], [13], [15], [16], [18], [51], [52], [54]. However, the endothelium can release an even more significant number of bioactive substances [39], [40].

7) Hormone Activation of Endothelial Luminal Receptors and the Associated Organ Parenchymal Response Maintains A Cause-Effect Relationship

When the luminal endothelial receptor activation by its hormone is the sole and sole stimulus triggering a parenchymal reaction, it defines a cause-effect relationship. This relationship demands that there is one and only one quantitative relationship between stimulus magnitude and cell response. The results in Fig. 8 support the concept of a cause-effect relationship. Seven AT1R agonists with different sizes were studied [17], [42] and given at a maximal equivalent concentration. The results of Ang II (1.05 kDa) and Ang II-Pols; $11 \mathrm{kDa}, 45 \mathrm{kDa}, 527 \mathrm{KDa}$, and 15,000 kDa are shown in Fig. 8.

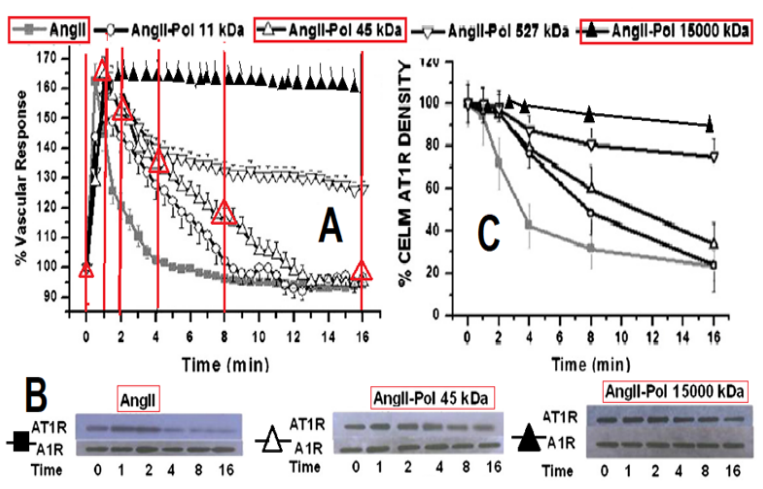

Fig. 8. Changes of vascular vasoconstriction (parenchymal response) and coronary endothelial luminal membrane (CELM) AT1R density as a result of a sustained separate administration of five agonists. The agonist infused were Ang II (1.03 kDa) or four Ang II-Pol's (11 kDa, $45 \mathrm{kDa}, 527 \mathrm{kDa}$, and $15,000 \mathrm{kDa}$ ). Fig. 8A. desensitization effects. Temporal course of coronary vascular vasoconstriction induced by sustained administration of either one of the five agonists used. Zero time marks the initiation of sustained agonis administration. Note that the responses rise, reach a maximum, followed by gradual decay that is slower as the size of the agonist increases. At times indicated by the straight red lines $(0,1,2,4,8$, and $16 \mathrm{~min})$, were sampled the amplitude of the responses and the CELM to determine AT1R density.

Fig. 8B. notice that the control loading protein, the adenosine receptor (A1R), remains unchanged. CELM Western blots of AT1R density changes during agonist treatment. Only shown are the results during treatment with $1.03 \mathrm{kDa}, 45 \mathrm{kDa}$, and 15,000 $\mathrm{kDa}$. The upper set of bands are the Western blots of AT1R densities at various times of treatment. Notice that for all agonists, as treatment proceeds, the band AT1R density decreases. Also, as the Ang II-Pol molecular weight rises, the decay of AT1R density is less. Fig. 8C. Receptor internalization. Temporal course of CELM AT1R density induced by sustained administration of Ang II (1.03 kDa, gray squares) or

Ang II-Pol's (11 kDa circles, $45 \mathrm{kDa}$ triangles, $527 \mathrm{kDa}$ upside-down triangles, and 15,000 kDa black triangles). CELM AT1R densities were determined at times indicated by red lines in $8 \mathrm{~A}$. Notice that as the size of

the agonist increases, there is a slower decay AT1R from its control

$(100 \%)$. Receptors internalized $=$ Control AT1R density $(100 \%)$ minus an AT1R density at a given time [17], [18].
- Response amplitude: A gradually growing response occurs at the initiation of a sustained administration of any agonist. All the responses reach the same maximum amplitude that slowly decays (desensitization). All response rates of decay induced by the different Ang IIPols vary, from the more rapid for Ang II to the slower as Ang II-Pol size increases (Fig. 8A). The rate of desensitization is inversely proportional to the Ang IIPol molecular weight.

- $\quad$ AT1 membrane receptor density: In Fig. 8A, during each Ang II-Pols administration, the red straight vertical lines mark the times $(0,1.2,4,8,16 \mathrm{~min})$, isolating the endothelial luminal membrane to determine the amount of AT1R protein by western-block analysis. Western Blot generates bands whose integrated density is proportional to the amount of protein (upper bars in Fig. 8B). During Ang II administration (Fig. 8B, left), AT1R band density gradually decays from the 0 to $16 \mathrm{~min}$ sample. This decay of AT1R band density is slower as the molecular weight of Ang II-Pol increases from 1.03 $\mathrm{kDa}$ to $15,000 \mathrm{kDa}$ (Fig. 8B). Fig. 8C shows the AT1R band density-time plots for 5 Ang II-Pols (kDa); 1.05 (Ang II), 11, 45, 527, and 15,000. At zero time (no hormone present), is taken as the AT1R control $100 \%$. The AT1R protein density decays as time proceeds because an increasing amount of AT1R is internalized (Fig. 8C). Internalization is more rapid for Ang II and is slower as Ang II-Pol size increases (Fig. 8C). The rates of internalization of AT1R and desensitization are inversely proportional to the molecular weight of the Ang II-Pol.

- Parenchymal response and CELM AT1R density maintain a unique quantitative relationship: The results with Ang II-Pol $45 \mathrm{kDa}$ (Fig. 8A and 8C). Take the values of parenchymal responses ([R], red triangles) at times indicated by the vertical red lines and the corresponding CELM AT1R densities ([AT1R] (Fig. $8 \mathrm{C})$. The plot [R] against [AT1R] yields a straight line (not shown). Similar plots for all the seven agonists yielded statistically the same straight line: $[\mathrm{R}]=\kappa$ [AT1R] $\kappa$ being a constant. Activation of a given [AT1R] always generates the same [R], a unique quantitative relationship; a cause-effect relationship [17], [18]. In addition, as previously suggested, the magnitude of the hormonal physiological response is determined by hormone receptor density [17], [46]-[50].

C. Lack of Hormone Exchange Between Circulatory and Interstitial Compartments, Summary of Diffusional and Functional Studies

The following facts support the proposal of the endothelial luminal layer being the primary regulator of parenchymal responses to circulating hormones:

1) Arterial and interstitial hormone concentration values in these two compartments differ significantly and are physically independent. The endothelial barrier minimizes free diffusional hormone exchange.

2) This intravascular-interstitial(s) compartmentation allows each compartment to regulate its hormone concentration independently.

3) The circulatory, endocrine action is restricted to luminal 
endothelial membrane receptors. Stoichiometrically, the endothelium releases distinct paracrine agent(s) that stimulates a particular parenchymal cell function.

4) The endothelium can release multiple paracrine agents, but in response to hormone stimulation, the release agent is site-dependent and selective.

5) Two hormonal stimuli control the parenchymal cell: An endothelial paracrine and an interstitial autocrine.

6) The circulating endocrine pool interacts with its parenchymal autocrine hormone pool solely through chemical signaling. The pools functionally likely are dependent on each other.

7) Endocrine activation of the luminal endothelial receptors is the first and sole site that triggers an organparenchymal response, a cause-effect relationship.

These functional and diffusional properties apply to Acetylcholine, Adenosine A1, A2, A3, Adrenergic- $\alpha 1-$, and $\beta 1$ and $\beta 3$, Bradykinin, Insulin, Vasopressin, Substance $\mathrm{P}$, Testosterone, Estradiol and Angiotensin II [2]-[7], [11]-[19], [23]-[26].

Activation of the luminal endothelial receptor by an endocrine hormone is the triggering mechanism of multiple sequential signaling processes in various compartments of an organ or/and various organs. The integration of these processes results in the physio-pathological response.

\section{AN UPDATED MODEL FOR ENDOCRINE ACTIONS ON ORGAN'S PARENCHYMA}

Fig. 9 shows a four-compartment model and simplified by excluding all intracellular compartments. At least three up to five compartments constitute an organ. In cases like the kidney and secretory glands, the tubules form the fourth compartment. This model is updated based on the experimental evidence presented above, contrasting with the classical shown in Fig. 1. The following seven descriptions hold for sustained endocrine administration within a 60minute range. The parenchymal-paracrine responses could be diverse cardiac inotropism, dromotropism, ionic channels permeability, vascular smooth muscle contraction, glomerular filtration rate, transmitter release from nerve end terminals, and release of hormones by glands:

1) The endothelial barrier physically separates at least three hormone compartments, the vascular endocrine, the interstitial(s), the autocrine, and the lymphatic. The source(s) of the autocrine may reflect a prolonged slow transport system of the endocrine or/and from or diverse endogenous synthesis systems.

2) The endothelial barrier and the epithelial barrier allow each compartment to regulate its hormone concentration independently.

3) These compartments are physically independent of each other but functionally are interdependent.

4) The endocrine hormone activates solely luminal endothelial receptors triggering the release of a specific paracrine agent.

5) In each compartment, the paracrine agent and autocrine hormones are the sole activators of the cells in that compartment.
6) The various cell types in these compartments functionally interact via chemical signaling, paracrinally, not by the mass exchange.

7) A lymphatic washout may regulate the levels of the interstitial hormone, and whether lymphatics is a source of signaling is unknow.

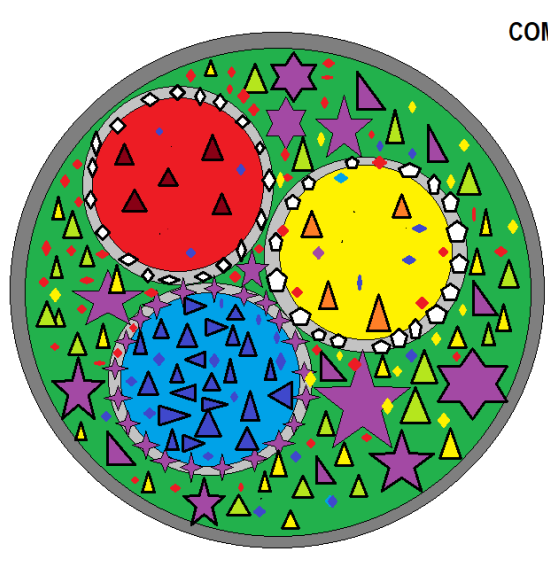

COMPARTMENTS AND THEIR HORMONE INTRAVASCLLAR $\triangle$ INTERSTITIAL $\triangle$ INTRPHATIC $\triangle$ PARACRINES RELEASE BY DIVERSE CELLS $\diamond \diamond$

\section{CELL TYPES:} ENDOTHELIAL $\bigcirc O$ EPITHELIAL \&े PARENCHYMAL

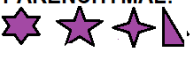
SAME HORMONE $\Delta=\Delta=\Delta=\Delta$

Fig. 9. This model a cross-section of a hypothetical organ with at least four compartments; 1) intravascular (red), 2) interstitial (green), intratubular (blue), and 3) lymphatic (yellow). These compartments may contain the same hormone, an endocrine $(\Delta)$ and autocrine; interstitial $(\Delta)$, intratubular $(\triangle)$, lymphatic $(\triangle)$. However, the hormone may have a different origin. In this model, the endocrine and autocrine hormones exchange very slowly across the endothelial, epithelial, and secretory cells that define their barrier. The lymphatic hormone may be a direct reflection of the interstitial hormone. The diverse paracrine agent's release by the various cells is represented by $\diamond \diamond$. The endocrine and autocrine hormones restrict their action to cells in their compartment. Upon endocrine activation, the endothelium releases paracrine agents that regulate processes of fibroblast, myocytes, nerve endings, epithelial and secretory cells. The Lymphatic circulation likely washes out the interstitial hormone and constitutes another functional compartment [2]-[7], [11]-[19], [21], [23]-[26], [34], [55]-[61].

However, in the case of endocrine-Ang II, this hormone triggers functional compartmental interactions, an integrated response. In this regard, studies in the kidney beautifully show five physical and physiological hormonal interacting compartments: intravascular, interstitial, tubular, intracellular, and lymphatic. Through selective autocrine and paracrine signaling, these compartments alter each other diverse functions; "the response." The integration of this biophysical compartmentalization is far from a clear understanding [59], [60], [62], [63], [64], [71], [72].

On the other hand, some responses require long-term (1015 days); chronic, hormone/antagonist administration. Ang II chronic administration causes sustained cardiac vascular vasoconstriction, hypertrophy, and fibrosis [55], [66]-[69], kidney afferent and efferent arterioles constriction, Ang II synthesis, tubular angiotensinogen mRNA, P2X's expression, plus other responses [32], [51]-[53], [59], [60], [70], [71]. A question remains unanswered if the effects induced by chronic vascular hormone administration result from the same endothelial paracrine agent(s) responsible for the short-term administration [13]. For example, ATP is a short-term paracrine mediator induced by Ang II in the heart and kidney. Nevertheless, chronic ATP receptor antagonists blocked sustained Ang II (14 days) administration effects on the kidney's glomerular hemodynamics and protein expression [51], [52]. This observation indicates that ATP is one of the responsible paracrine mediators for Ang II's long- 
term and short-term actions. Thus, it is likely that this possibility also applies to other hormones.

\section{New Research AND Therapeutic Challenges}

Organ compartments: vascular, interstitials; tubular and lymphatic, are physically separated, contain different concentrations of the same hormone (endocrine and autocrine), and are paracrinally interdependent. Chronic therapeutic administration of the circulating hormone or receptor blockers triggers the various interstitial compartment's functional, chemical, and structural changes whose integration defines the overall organ response. Taken as organ examples the heart or kidney:

1) In vivo, the vascular endocrine, without o minimal access to the parenchyma, the sole and direct triggering action reside on the luminal endothelial membrane. Moreover, along the vascular tree, the endocrine causes the release of various endothelial paracrine agents that are site and function-specific, and most of them are unknown.

2) However, associated with intravascular receptor hormone activation or blockade, functional, chemical, and structural changes in the interstitial compartments occur. Are these changes the response of the specific paracrine agent alone or the combination autocrineparacrine agent?

Despite all these uncertainties, experimentally and clinically are administered hormones or antagonists to achieve an effect. However, the final result may differ between individuals that required personalized therapeutics, actions in a specific compartment. That is to selectively restrict the access of the hormone, its antagonist, or the selective paracrine agent involved [59], [60]. For that, we have to design site-specific agonists and antagonists and identify the involved paracrine agents [62], [63].

The research and therapeutic challenges to achieve a looked-for pharmacological effect are:

a. To regulate the degree of barrier properties of the endothelial wall and other wall compartments for agonist and their blockers [34], [61], [73] selectively.

b. To identify the paracrine agents involved and their antagonists in every functional compartment.

c. To find ways for the agonist/antagonist to gain exclusive access to the target compartment,

d. To describe the particular properties of the target hormone receptor in that compartment and chemically design the agonist or antagonist with the highest possible specificity.

\section{CONCLUSION}

The vascular network is so dense that virtually every organism cell has several vascular endothelial cells as immediate neighbors. Consequently, more than one glandular-endothelial cell per parenchymal cell constitutes the anatomical foundation for a powerful, confined, and efficient regulatory system. Furthermore, solid experimental evidence supports the proposal of the endothelial luminal layer being the sole site of action of blood hormones. The current knowledge requires the incorporation of this new concept, a new paradigm.

\section{ACKNOWLEDGMENT}

I would like to thank Dr. Guillermo Ceballos and Dr. Dr. Nayelli Najera for their assistance and valuable suggestions. Dr. Ceballos and Najera are from the Seccion de Estudios de lnvestigacion y Posgrado, Escuela Superior de Medicina, Instituto Politecnico Nacional. Mexico.

\section{REFERENCES}

[1] Berthold AA. Lehrbuch der Phsiologie des Menschen und der Thiere (1 ed.). Göttingen: Vandenhoek und Ruprecht; 1829.

[2] Armstrong KJ., Noall MW., Stouffer JE. Dextran-linked insulin: a soluble high molecular weight derivative with biological activity in vivo and in vitro. Biochem. Biophys. Res. Commun. 1972; 47: 354-360.

[3] Balcells E., Suarez J., Rubio R. The functional role of intravascular coronary endothelial adenosine receptors. Eur. J. Pharmacol. 1992; 210: 1-9.

[4] Balcells E., Suarez J., Rubio R. Implications of the coronary vascular endothelium as a mediator of the vasodilatory and dromotropic actions of adenosine. J. Mol. Cell. Cardiol. 1993; 25: 693-706.

[5] Ceballos G., Rubio R. Endothelium-mediated negative dromotropic effects of intravascular acetylcholine. Eur. J. Pharmacol. 1988; 362: $157-166$.

[6] Rubio R., Ceballos G., Balcells E. Intravascular adenosine: the endothelial mediators of its negative dromotropic effects. Eur $J$ Pharmacol. 1999; 370: 27-37.

[7] Rubio R., Ceballos C. Functional implications of sole and selective activation of intravascular coronary endothelial hormonal receptors. Acta Pharmacologica Sinica. 2000; 21: 577-586.

[8] Nees S., Herzog V., Becker BF., Bock M., Des Rosiers C., Gerlach E. The coronary endothelium: a highly active metabolic barrier for adenosine. Res. Cardiol. 1985; 80: 515 .

[9] Olsson RA., Davis CJ., Khouri EM., Paterson ER. Evidence for an adenosine receptor on the surface of dog coronary myocytes. Circ. Res. 1976; 39: 93-98.

[10] Schrader J., Nees S., Gerlach E. Evidence for a cell surface adenosine receptor on coronary myocytes and atrial muscle cells. Pflueger's Arch. 1977; 369: 251-257.

[11] Bevan JA., Duckles SP. Evidence for alpha-adrenergic receptors on intimal endothelium. Blood Vessels 1975; 12: 307-310.

[12] Castillo-Hernández J., Rubio R., Maldonado-Cervantes MI. A limited vs a transient AT1R internalization indicates that distinct mechanisms of AT1R activation are possible. Faseb. 2021; 35: 2021.

[13] Castillo-Hernandez JR., Rubio-Gayosso I., Sada-Ovalle I., GarciaVazquez A., Ceballos G., Rubio R. Intracoronary Angiotensin II causes inotropic and vascular effects via different paracrine mechanisms. Vasc. Pharmacol. 2004; 41: 147-158.

[14] Castillo-Hernandez JR., Torres-Tirado D., Barajas-Espinosa A., ChiAhumada E., Ramiro-Diaz J., Ceballos G., et al. Two dissimilar AT1 agonists distinctively activate AT1 receptors located on the luminal membrane of the coronary endothelium. Vasc. Pharmacol. 2009; 51 314-322.

[15] Ceballos G., Figueroa L., Rubio I., Gallo G., Garcia A., Martinez A., et al. Acute and nongenomic effects of testosterone on isolated and perfused rat heart. J Cardiovasc Pharmacol. 1999; 33: 691-7.

[16] Rubio R., Ceballos G., Sole activation of three luminal adenosine receptor subtypes in different parts of the coronary vasculature. Am. J. Physiol. Heart Circ. Physiol. 2003; 284: H204-H214.

[17] Rubio R., Torres-Tirado D., Castillo-Hernández J., Chi-Ahumada E., Ramiro-Diaz J. The coronary endothelium behaves as a selective functional diffusion barrier for intravascular Angiotensin II. Vasc. Pharmacol. 2013; 58: 54-63.

[18] Torres-Tirado D., Ramiro-Diaz J., Knabb M., Rubio R. The molecular weight of different angiotensin II polymers directly determines the density of endothelial membrane receptors and coronary vasoconstriction. Vasc. Pharmacol. 2013; 58: 346-355.

[19] Zenteno ST., Ceballos G., Rubio R. Effects of arginine vasopressin in the heart are mediated by specific intravascular endothelial receptors. Europ. J. Pharmacol. 2000; 410: 15-23.

[20] Dostal DE., Baker KM. The Cardiac Renin-Angiotensin System; Conceptual, or a Regulator of Cardiac Function? Circ Res. 1999; 85: 643-650. 
[21] Nyui N., Tamura K., Mizuno K., Ishigami T., Hibi K. Strecth-induced MAP kinase activation in cardiomyocytes of angiotensinogen-deficient mice. Biochem and Biophysc Res Comm. 1997; 235: 36-41.

[22] Salas M., Vila-Petroff M., Palomeque J., Aiello E., Mattiazzi A. Positive inotropic and negative lusitropic effect of angiotensin II: intracellular mechanisms and second messengers. J. Mol. Cell. Cardiol. 2001; 33: 1957-1971.

[23] Figueroa-Valverde L., Luna H., Castillo-Henkel C., Munoz-Garcia O., Morato-Cartagena T., Ceballos-Reyes G. Synthesis and evaluation of the cardiovascular effects of two, membrane impermeant, macromolecular complexes of dextran-testosterone. Steroids. 2002; 67: 611-619.

[24] Rodriguez-Hernandez A., Rubio-Gayosso I., Ramirez I., Ita-Islas I., Meaney E., Gaxiola S. et al. Intraluminal-restricted 17ß-estradiol exerts the same myocardial protection against ischemia/reperfusión injury in vivo as free 17ß-estradiol. Steroids. 2008; 73: 528-538

[25] Sierra-Ramirez A., Morato T., Campos R., Rubio I., Calzada C., Mendez E., et al. Acute effects of testosterone on intracellular $\mathrm{Ca} 2+$ kinetics in rat coronary endothelial cells are exerted via aromatization to estrogens. Am J Physiol. 2004; 287: H63-71.

[26] Kawada T., Yamazaki T., Akiyama T., Li M., Zheng C., Shishido T., Mori H., Sugimachi M. Angiotensin II attenuates myocardial interstitial acetylcholine release in response to vagal stimulation. $\mathrm{Am}$. J. Physiol. 2007; 293: H2516-H2522.

[27] Dell'Itali LJ., Meng QC., Balcells E., Wei CC., Palmer R., Hageman GR., Durand J., Hankes GH., Oparil S. Compartmentalization of angiotensin II generation in the dog heart. Evidence for independent mechanisms in intravascular and interstitial spaces. J. Clin. Invest. 1997; 100: 253-258

[28] Gidday JM., Rubio R., Berne RM. Increases in coronary flow by intracoronary adenosine occur independently of changes in interstitial fluid adenosine. Physiologist. 1987; 30: 187.

[29] Gidday JM., Hill HE., Rubio R., Berne RM. Estimates of left ventricular interstitial fluid adenosine during catecholamine stimulation. Am. J. Physiol. 1988; 254: H207-H216.

[30] Heller L., Morman D. 1988, Estimates of interstitial adenosine from surface exudates of isolated rat hearts. J. Mol. Cell. Cardiol. 1988; 20: 509

[31] Lasley RD., Hegge JO., Noble MA., Mentzer RM. Comparison of interstitial fluid and coronary venous adenosine levels in vivo porcine myocardium. J. Mol. Cell. Cardiol. 1998; 30: 1137-1147.

[32] Navar LG., Kobori H., Prieto MC., Gonzalez-Villalobos RA. Intratubular Renin-Angiotensin System in Hypertension. Hypertension. 2011; 57: 355-362.

[33] Akira N., Seth DM., Navar LG. Renal Interstitial Fluid Concentrations of Angiotensins I and II in Anesthetized Rats. Hypertension. 2020; 39 : 129-134.

[34] Rafael R., Knabb M. The luminal endothelial membrane-glycocalyx; functionalities in health and disease. Morgan and Claypool, Science Publishers; 2017

[35] Siragy HM., Howell NL., Ragsdale NV., Carey RM. Renal interstitial fluid angiotensin. Modulation by anesthesia, epinephrine, sodium depletion, and renin inhibition. Hypertension. 1995; 25: 1021-1024.

[36] Tietjan CS., Tribble CG., Gidday JM., Philips CM., Belardinelli L. Rubio R., Berne RM. Interstitial adenosine in guinea pig hearts: an index obtained by epicardial disks. Am. J. Physiol. 1990; 259: H1471H1476.

[37] Chih-Chang W., Lucchesi PA., Tallaj J., Wayne E. Bradley WE. Powell PC., Dell'Italia LJ. Cardiac interstitial bradykinin and mast cells modulate the pattern of LV remodeling in volume overload in rats. Am J Physiol. 2003; 285: H784-H792.

[38] Furchgott R., Zawadzki, J. The obligatory role of endothelial cells in the relaxation of arterial smooth muscle by acetylcholine. Nature. 1980; 288: 373-376.

[39] Frangos JA. Flow effects on endothelial cell signal transduction, function, and mediator release. New York: Oxford Univ. Press; 1995.

[40] White CR., Frangos JA. The shear stress of it all: The cell membrane and mechanochemical transduction. Phil Trans $R$ Soc. 2007; 362: 1459-1467.

[41] Taylor AE., Granger DN. Exchange of macromolecules across the microcirculation. Handbook of Physiology; 1984.

[42] Haga, K., Haga, T. Affinity chromatography of the muscarinic acetylcholine receptor. J. Biol. Chem. 1983; 258: 13575- 13579.

[43] Arroyo-Flores B., Chi-Ahumada E., Erika Briones-Cerecero E., Barajas-Espinosa A., Perez-Aguilar S., et al. Cardiac Ischemia and Ischemia/Reperfusion Cause Wide Proteolysis of the Coronary Endothelial Luminal Membrane: Possible Dysfunctions. The Open Cardiovascular Medicine Journal. 2011; 5: 001-007

[44] Perez-Aguilar S., Torres-Tirado D., Martell-Gallegos G., VelardeSalcedo J., Barba-de la Rosa A. P., Knabb M., et al. G protein-coupled receptors mediate coronary flow- and agonist-induced responses via lectin-oligosaccharide interactions. Am J Physiol Heart Circ Physiol. 2014, 306: H699-H708.

[45] Torres-Tirado D., Knabb M., Castaño I., Patrón-Soberano A., De Las Peñas A., Rubio R. Candida glabrata binds to glycosylated and lectinic receptors on the coronary endothelial luminal membrane and inhibits flow sense and cardiac responses to agonists. Am J Physiol. 2016; 310 : R24-R32.

[46] De Godoy MA., Rattan S. Translocation of AT1- and AT2 receptors by higher concentrations of angiotensin II in the smooth muscle cells of rat internal anal sphincter. J. Pharmacol. Exp. Ther. 2006; 319: 10881095.

[47] Drake MT., Shenoy SK., Lefkowitz RJ. Trafficking of G proteincoupled receptors. Circ. Res. 2006; 99: 570-582.

[48] Koening J., Edwardson M. Endocytosis and recycling of G proteincoupled receptors. Trends Pharmacol. Sci. 1997; 18: 276-287.

[49] Olivares-Reyes AJ., Smith RD., Hunyady L., Shah BH., Catt KJ. Agonist-induced Signaling, Desensitization, and Internalization of a Phosphorylation-deficient AT1A Angiotensin Receptor. L. Biol. Chem. 2001; 276: 37761-37768.

[50] Toth D., Toth J., Gulyas G., Balla A., Balla T., Hunyady L., Varnai P. Acute depletion of plasma membrane phosphatidylinositol 4,5bisphosphate impairs specific steps in endocytosis of the G-protein coupled receptor. J. Cell Sci. 2012; 125: 2185-2197.

[51] Franco M., Bautista-Pérez R., Cano-Martínez A., Pacheco U., Santamaría J., del Valle Mondragón L., et al. Physiopathological implications of P2X1 and P2X7 receptors in regulation of glomerular hemodynamics in angiotensin II-induced hypertension. Am J Physiol Renal Physiol. 2017; 313: F9 -F19.

[52] Graciano ML., Nishiyama A., Jackson K., Seth DM., Ortiz RM., PrietoCarrasquero M., et al. Purinergic receptors contribute to early mesangial transformation and renal vessel hypertrophy during angiotensin II-induced hypertension. Am J Physiol. 2008; 294: F161F169.

[53] Ji X., Naito Y., Hirokawa G., Weng H., Hiura Y., Takahashi R., et al. $\mathrm{P} 2 \mathrm{X}(7)$ receptor antagonism attenuates hypertension and renal injury in Dahl salt-sensitive rats. Hypertens Res. 2012; 35: 173-179.

[54] Larner J., Allan G., Kessler C., Reamer P., Gunn R., Huang LC. Phosphoinositol glycan derived mediators and insulin resistance. Prospects for diagnosis and therapy. J Basic Clin Physiol Pharmacol. 1998; 9: 127-137.

[55] Booz DW., Dostal DE., Baker KM. Paracrine actions of cardiac fibroblasts on cardiomyocytes: implications for the cardiac reninangiotensin system. Am. J. Cardiol. 1999; 83: 44-47.

[56] Chiu JD., Richey JM., Harrison LN., Zuniga E., Kolka CM., Kirkman E., et al. Direct Administration of Insulin into Skeletal Muscle reveals That the Transport of Insulin Across the capillary endothelium Limits the Time Course of Insulin o Activate Glucose Disposal. Diabetes. 2008; 57: 828-833.

[57] Kolka CM. The vascular endothelium plays a role in insulin action. Clin Exp Pharmacol Physiol. 2020; 47: 168-175.

[58] Kolka CM., Bergman RN. The Barrier Within Endothelial Transport of Hormones. Physiology. 2012; 27: 237-247.

[59] Schnermann J. Concurrent Activation of Multiple Vasoactive Signaling Pathways in Vasoconstriction Caused by Tubulo-glomerular Feedback: A Quantitative Assessment. Ann Rev Physiol. 2015; 77:301322.

[60] Schnermann J., Levine DZ. Paracrine factors in tubule-glomerular feedback: adenosine, ATP, and nitric oxide. Ann Rev Physiol. 2003; 65: 501-529

[61] Stolwijk JA., Zhang X., Gueguinou M., Zhang W., Matrougui K. Regulation of endothelial barrier function. J Biol Chem. 2016; 291 : 22894-22912.

[62] Speth RC., Giese MJ. Update on the Renin-Angiotensin System. $J$ Pharmacol, \& Clinic. Toxicol. 2013; I: 1004-1017

[63] Li XC., Wang CH., Leite A. P. O., Zhuo J. L. Intratubular, Intracellular, and Mitochondrial Angiotensin II/AT 1 (AT1a) Receptor/NHE3 Signaling Plays a Critical Role in Angiotensin II-Induced Hypertension and Kidney Injury. Front. Physiol. 2021; 12: 702797.

[64] De Mello, WC., Danser, AHJ. Angiotensin II and the heart: on the intracrine renin-angiotensin system. Hypertension. 2000; 35: 1183 1188.

[65] Dobson, JG. Reduction by adenosine of the isoproterenol-induced increase in cyclic adenosine 3',5'-monophosphate formation and glycogen phosphorylase activity in rat muscle. Circ. Res. 1978; 43: 785.

[66] Berk, BC. Angiotensin II signal transduction in vascular smooth muscle: pathways activated by specific tyrosine kinases. J. Am. Soc. Nephrol. 1999; (Suppl. 11), S62-S68. 
[67] Ishida M., Ishida T., Thomas SM., Berk BC. Activation of extracellular signal-regulated kinases (ERK1/2) by angiotensin II is dependent on cSrc in vascular smooth muscle cells. Circ. Res. 1998; 82: 7-12.

[68] Marrero MB., Schieffer B., Paxton WG., Heerdt L., Berk BC. Delafontaine, P., Bernstein KE. Direct stimulation of JAK/STAT pathway by the Angiotensin AT1 receptor. Nature. 1995; 375: $247-$ 250.

[69] Touyz RM., El Mabrouk M., He G., Wu XH., Schiffrin EL., Mitogenactivated protein/extracellular signal-regulated kinase inhibition attenuates angiotensin II-mediated signaling and contraction in spontaneously hypertensive rat vascular smooth muscle cells. Circ. Res. 1999; 84: 505- 515.

[70] van Kats JP., de Lannoy LM., Danser AHJ., van Meegen JR., Verdouw PD., Schalekamp MADH. Angiotensin II type (AT1) receptormediated accumulation of Angiotensin II in tissues and its intracellular half-life in vivo. Hypertension. 1997; 30: 42- 49.

[71] Li XC, Carretero OA., Navar LG., Zhuo JL. AT1 receptor-mediated accumulation of extracellular angiotensin II in proximal tubule cells: role of cytoskeleton microtubules and tyrosine phosphatases. $\mathrm{Am} \mathrm{J}$ Physiol. 2006; 291: F375-F383.

[72] Ziganshin AU, Khairullin AE, Hoyle CH., Grishin SN. Modulatory Roles of ATP and Adenosine in Cholinergic Neuromuscular Transmission. Int. J. Mol. Sci. 2020; 21, 6423.

[73] Volpe E., Battistini L., Borsellino G. Advances in T Helper 17 Cell Biology: Pathogenic Role and Potential Therapy in Multiple Sclerosis. Mediators of Inflammation. 2015 